\title{
Effect of Coating Thickness on a Solid-Liquid Two-Phase Flow Centrifugal Pump under Water Medium
}

\author{
Kaikai Luo ${ }^{1,2}$ - Yong Wang1,* - Houlin Liu ${ }^{1}$ - Matevž Dular ${ }^{2}$ - Jie Chen ${ }^{1}-$ Zilong Zhang $^{1}$ \\ 1 Jiangsu University, Research Center of Fluid Machinery Engineering and Technology, China \\ 2 University of Ljubljana, Faculty of Mechanical Engineering, Slovenia
}

Spraying a coating on the surface of wet parts of a solid-liquid two-phase flow centrifugal pump could effectively increase its service life. To research the effect of coating thickness on the performance of the pump, a solid-liquid two-phase flow pump with a speed of $n_{s}=81.5$ was chosen, and polyurethane coatings with different thicknesses were sprayed on the surface of the blades of the impeller. The influence of coating thickness on the performance of the pump was tested under the condition of a water medium. Combined with numerical simulation, the internal flow, pressure fluctuations and radial force of the model pump were further analysed in detail. Keeping the blade outlet angle unchanged, the head and efficiency of the pump decrease with the increase of the coating thickness, and the amplitude of the decrease increases with the increase of the coating thickness. The peak value of pressure fluctuations increases with the increase of coating thickness. The pressure value on the impeller inlet increases first and then decreases with the increase of coating thickness. Radial force of impeller increases with the increase of coating thickness, and the radial force distributes in the triangle, which is the same as the number of blades.

Keywords: coating thickness, solid-liquid two-phase flow pump, numerical simulation, pressure fluctuation, radial force

Highlights

- A significant agreement of results under experiment and numerical simulation are captured. Additionally, the relative deviation of the head and efficiency at each flow condition is within $5 \%$.

- The outlet pressure pulsation is concentrated in the low-frequency region, and the main peak value of the pressure fluctuation is at the blade frequency.

- $\quad$ There is a large area of low pressure at each inlet of the impeller. As the coating thickness increases, the pressure at the impeller inlet increases first and then decreases.

- With the increase of the coating thickness, the streamline in the channel becomes increasingly inhomogeneous, and the streamline in the runner changes from uniform distribution to distribution near the impeller pressure face.

- $\quad$ The radial force of the impeller increases with the increase of the coating thickness, and the radial force of the impeller is distributed symmetrically in a triangle, which is the same as the number of blades.

\section{O INTRODUCTION}

Solid-liquid two-phase flow centrifugal pumps, indispensable equipment for solid-liquid mixed medium transportation in production and daily life, play an essential role in metallurgy, water conservation, and mining. Due to the specifics of operation conditions, the solid-liquid mixture will cause wear-and-tear damage on the wet parts of a solid-liquid two-phase flow centrifugal pump, shorten the service life, and reduce operation stability. The wear of wet parts has great influence on pump performance, such as head and efficiency reduction, vibration intensification, etc.

Research studies have been made on the characteristics of erosion caused by solid-liquid flow in pumps.

Noon and Kim [1] conducted three-dimensional numerical analysis for the prediction of erosion on the head and efficiency losses for lime slurry flow through centrifugal pumps and found that the temperature effect is critical for erosion damage. Lai et al. [2] employed the Eulerian-Lagrangian approach and combined it with the Grant and Tabakoff particlewall rebound model to predict particle behaviours and erosion wear in the centrifugal pump. The results show that with the increase of the concentrations of solid particles, the frequency of impingement and rebound will increase. The most serious erosion regions are around the middle of the hub and the trailing edge of blades pressure side. Sharma [3] carried out systematic studies of the erosion wear in a centrifugal slurry pump. The results show that the blade erosion rate is 10 times greater than the hub erosion rate. Compared with other parts of the impeller, the erosion rate near the leading edge is more severe. Bross and Addie [4] developed a simple model and predicted the influence of the different parameters on the wear behaviour of an impeller nose in the centrifugal slurry pump. The results show that the wear of the impeller and the liner will decrease with the increase of pressure coefficient and clearing vane height. 
In addition, some scholars researched the performance of solid-liquid flow in pumps. Wu et al. [5] researched three-dimensional unsteady flow characteristics in the centrifugal slurry pump for solid-liquid flow. The results show the fluctuation of the pressure and flow velocity, particularly near the volute tongue. The fluctuations of the head and radial forces are $8.1 \%$ and $85.7 \%$, respectively. Liquids are more influenced by the clocking effect than solids are. Shi et al. [6] designed a new experimental facility without agitation for an internal solid-liquid two-phase flow test in a centrifugal pump with PIV. Ning and Wang [7] studied the solid-liquid mixed flow hydraulic characteristics and turbulent kinetic energy distribution in centrifugal pumps. Wang and Qian [8] found that the head and shaft power decrease as the silt concentration and silt particle size increase for a double-section pump with experimental methods. Mishra and Ein-Mozafari [9] analysed the performance of the Maxblend impeller in solidliquid operation using the Eulerian-Eulerian method, standard $k-\varepsilon$ turbulence model and sliding mesh technique. Cando et al. [10] adopted the filter-based RANS $(k-\varepsilon)$ and Eulerian-Lagrangian method to analyse unsteady liquid-solid flow around a step in a rectangular channel, and their results revealed that the vorticity described by the Q-criterion promotes the particle motion at the wake area. Zhao et al. [11] researched the effect of the diameter size of particles and concentration on external performance, pressure and turbulent kinetic energy distribution.

Generally, coating material with better wear resistance, including epoxy resin mortar coatings [12] to [14], composite nylon coatings [15], polyurethane coatings [16] and other polymer coatings [17], sprayed on the surface of wet parts, is used to deal with the problem of the wear and tear of solid-liquid two-phase flow pumps. After spraying the coating, parameters such as the thickness and wrapping angle of pump impeller are changed, which has a significant influence on pump performance. It is necessary to focus on the effect of coating thickness on pump performance.

In this work, a solid-liquid two-phase flow pump with a specific speed $n_{\mathrm{s}}=81.5$ was chosen as the research subject. The surface of the impeller of the pump was sprayed with polyurethane coatings of different thickness to research the effect of coating thickness on the performance of the model pump.

\section{RESEARCH SUBJECT, SCHEME AND TEST SYSTEM}

\subsection{Research Subject}

A two-phase flow centrifugal pump with cylindrical blades was chosen as the research subject, whose nominal flow rate $Q_{\mathrm{n}}=20 \mathrm{~m}^{3} / \mathrm{h}$, head $H=22 \mathrm{~m}$ and efficiency $\eta=48 \%$. The main parameters of the model pump are summarized in Table 1, and its photo is shown in Fig. 1.

Table 1. Main parameters of the model pump

\begin{tabular}{lc}
\hline Specific speeds & $n_{s}=81.5$ \\
\hline Nominal flow rate & $Q_{\mathrm{n}}=20 \mathrm{~m} 3 / \mathrm{h}$ \\
\hline Rotational speed & $n=2900 \mathrm{r} / \mathrm{min}$ \\
\hline Impeller inlet diameter & $D_{1}=65 \mathrm{~mm}$ \\
\hline Impeller outlet diameter & $D_{2}=160 \mathrm{~mm}$ \\
\hline Outlet impeller width & $b=7 \mathrm{~mm}$ \\
\hline Thickness of blade & $\delta=6 \mathrm{~mm}$ \\
\hline Number of blades & $z=3$ \\
\hline Base diameters of volute & $D_{3}=182 \mathrm{~mm}$ \\
\hline Inlet width of volute & $b_{3}=26 \mathrm{~mm}$ \\
\hline Diffuser outlet diameter & $D_{4}=50 \mathrm{~mm}$ \\
\hline
\end{tabular}

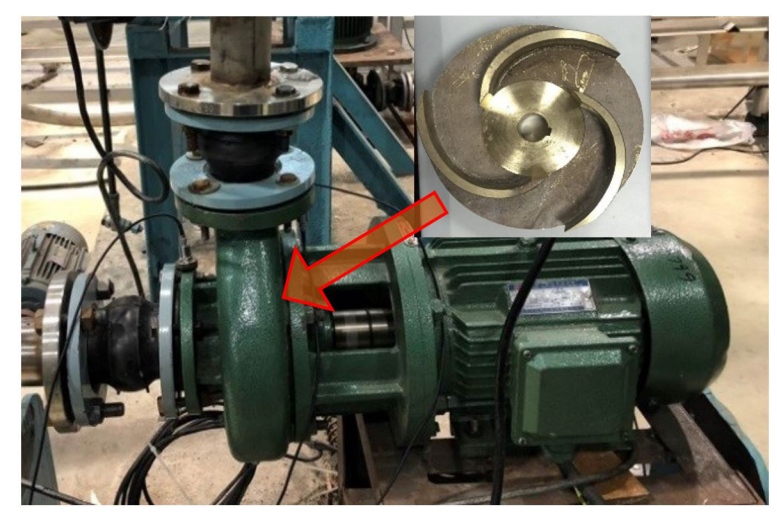

Fig. 1. Photo of the model pump with a cylindrical blade

\subsection{Test Rig and Measuring Point Distribution}

The test system included the model pump, pressure fluctuation testing system, electromagnetic flowmeter and power supply control system, and other features. The pump performance was tested in the test rig schematized in Fig. 2a. The water was pumped from and returned to a $10 \mathrm{~m}^{3}$ reservoir. The shaft torque and rotational speed were monitored by a torque and speed sensor with an error rate of $\pm 0.10 \%$. Static pressure values were measured at the inlet and outlet of the pump by a differential pressure transfer, and the uncertainty was within $\pm 0.10 \%$. The flow rate was 

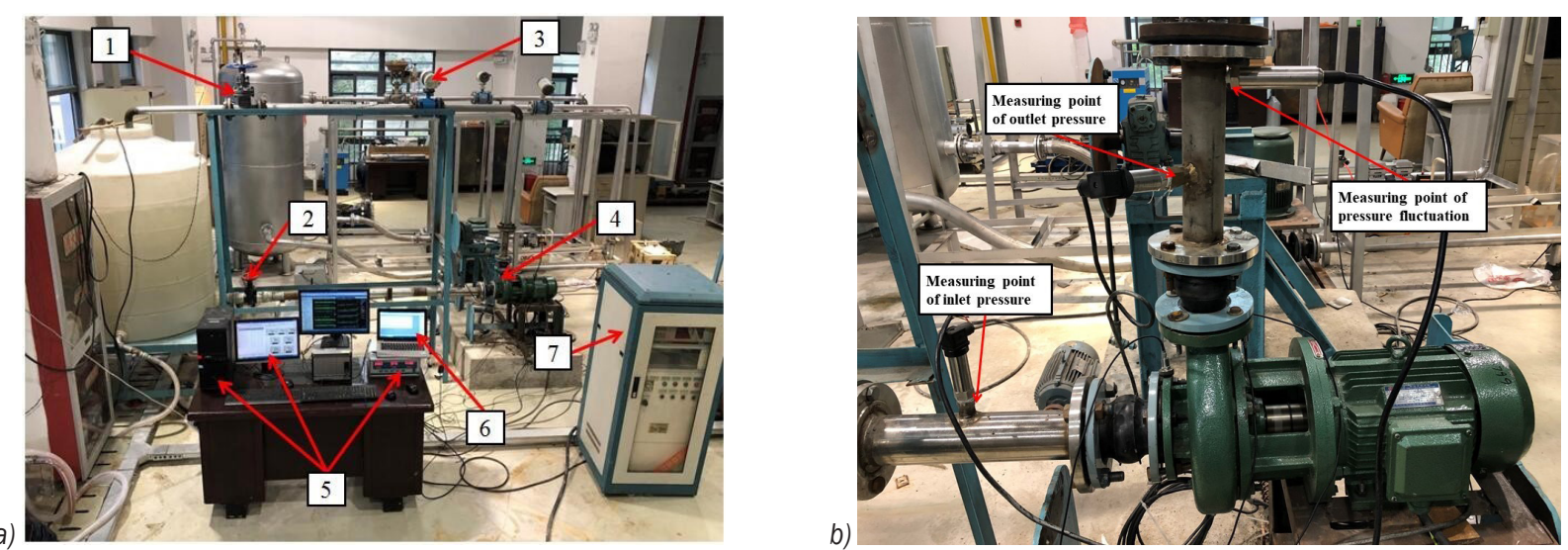

Fig. 2. Test system and measuring point distribution; a) test system of the model pump: 1. outlet valve, 2. inlet valve, 3. electromagnetic flowmeter, 4. model pump, 5. performance test system, 6. pressure fluctuation testing system, 7. power control system;

b) distribution of measuring point on static pressure and pressure fluctuation

measured by using a magnetic flow meter with an uncertainty of $\pm 0.14 \%$. The measurement accuracy of pump efficiency was quantified as $\pm 0.30 \%$.

The inlet and outlet pressure measurement points were set at twice the diameter of the inlet and outlet flanges. The pressure fluctuation measurement point was set at 4 times the outlet diameter of the outlet flange. The distribution of measurement points is shown in Fig. 2b.

\subsection{Scheme}

Non-dimensional thickness of coating thickness was dealt with to analyse the effect caused by coating thickness expediently, whose ratio was defined as $K_{i}$
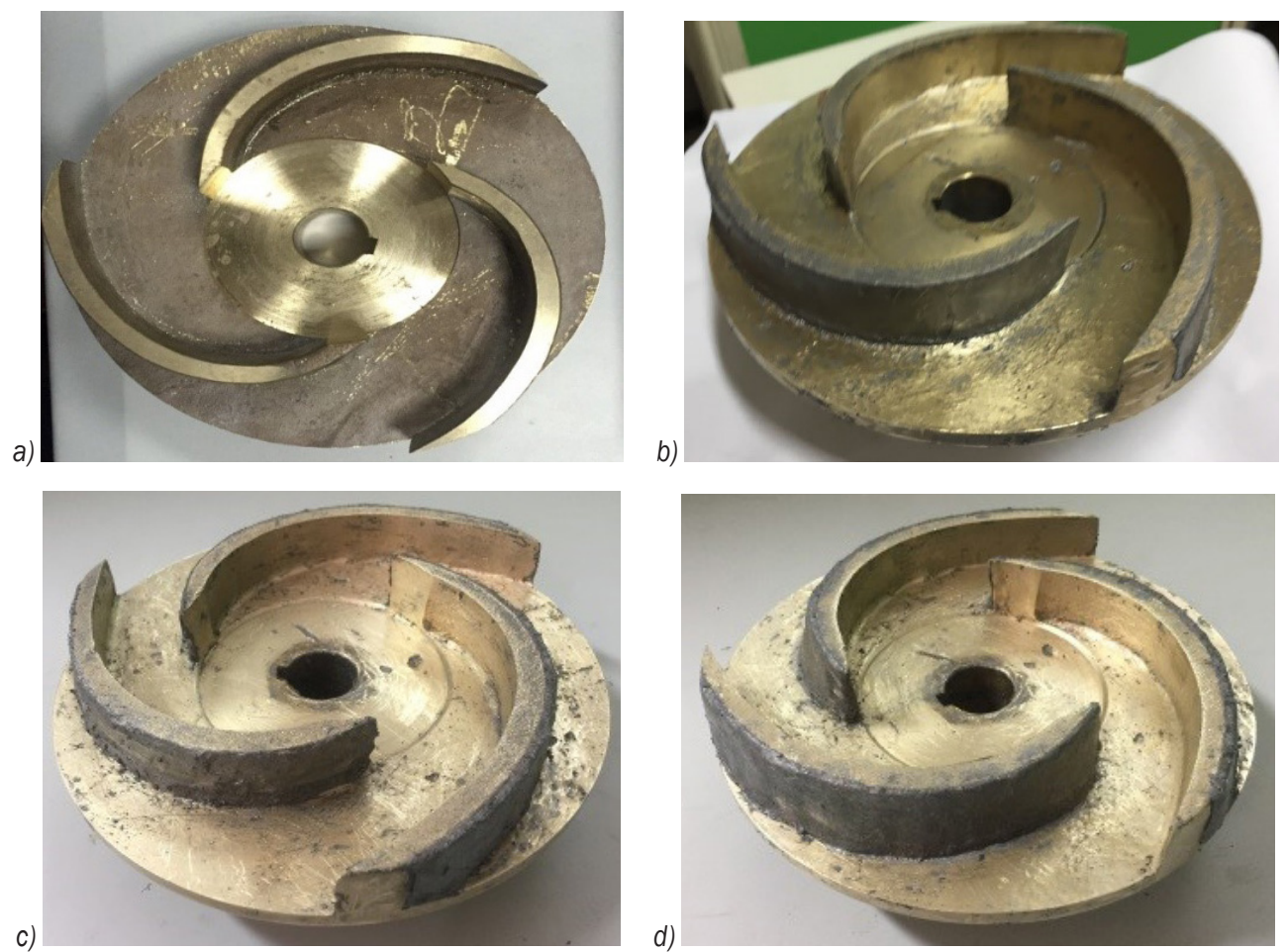

Fig. 3. Coating sprayed on impeller; a) coating thickness ratio $K_{0}$; b) coating thickness ratio $K_{1}$, c) coating thickness ratio $K_{2} ;$ d) coating thickness ratio $K_{3}$ 


$$
K_{i}=\frac{\delta_{i}}{\delta},
$$

where $K_{i}$ is the ratio of coating thickness. $\delta_{i}$ represents coating thickness, $(i=0,1,2,3)$, and $\delta$ is the thickness of the blade.

The polyurethane material with better comprehensive properties was sprayed on the pressure surface of the impeller. The ratio of coating thickness is $K_{0}=0\left(\delta_{0}=0 \mathrm{~mm}\right), K_{1}=1 / 6\left(\delta_{1}=1 \mathrm{~mm}\right), K_{2}=1 / 3$ $\left(\delta_{2}=2 \mathrm{~mm}\right)$ and $K_{3}=1 / 2\left(\delta_{3}=3 \mathrm{~mm}\right)$. The pictures of coating sprayed on impeller is shown in Fig. 3.

\section{COMPUTATION MODEL AND NUMERICAL SIMULATION}

\subsection{Computation Model}

The computation model, including the impeller, volute, inlet extension and outlet extension, was built with Creo 3.0 commercial software. The purpose of adding an extension of the inlet and outlet is to reduce velocity gradient and improve simulation accuracy. The computational domain of model pump with $K_{0}$ is shown in Fig. 4.

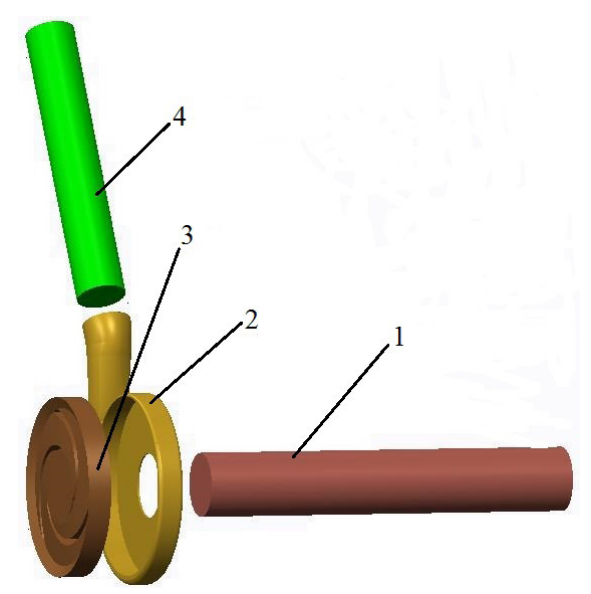

Fig. 4. Computational domain of model pump with $K_{0}$; 1 inlet extension; 2 volute 3 impeller; 4 outlet extension

\subsection{Computational Domain Discretization and Mesh Sensitivity Analysis}

The fluid domain of the model pump was discretized by the commercial software ANSYS ICEM. To guarantee fluid development enough, the length of the inlet extension is 5 times the inlet diameter of the centrifugal pump, and the outlet extension length is 10 times longer than the outlet diameter of the volute. Unstructured tetrahedral cells were used to discretize the computational domain. Computational model grids of the model pump are shown in Fig. 5.

A detailed mesh sensitivity analysis of the computational domain was performed to eliminate the influence of the mesh factor. The same topological structure of the computational domain was employed for the research model. Seven schemes of grids were obtained at the same premise of mesh quantity. The numbers of grids for seven cases were 975,000, $1,203,000, \quad 1,415,000, \quad 1,694,000, \quad 1,981,000$, $2,228,000$ and $2,602,800$.

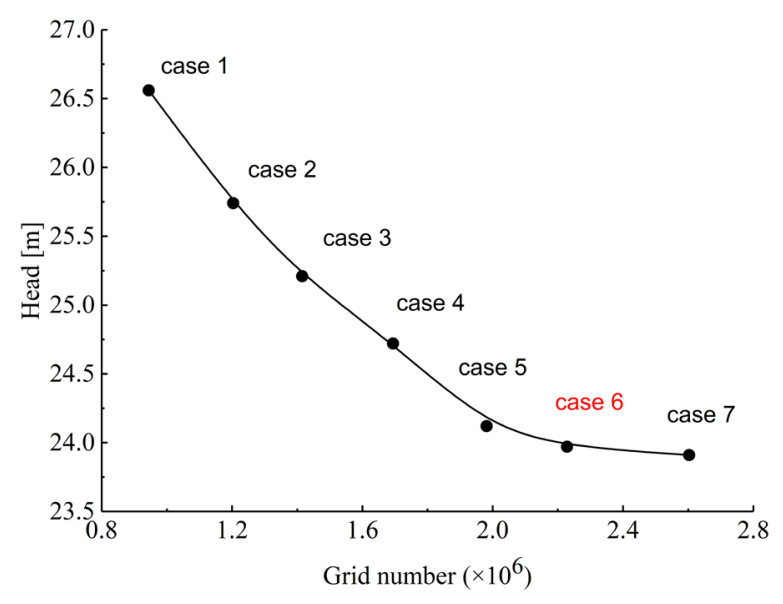

Fig. 6. Mesh sensitivity analysis

The head value was taken as the criterion to evaluate the mesh sensitivity. As can be observed in

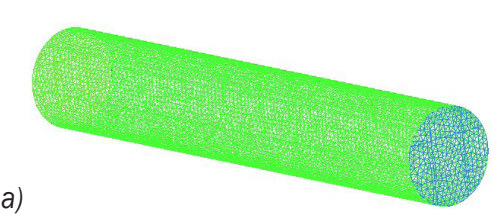

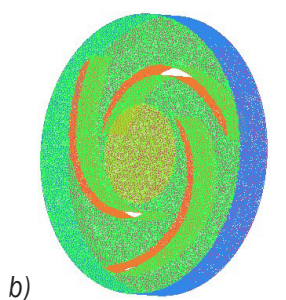

c)

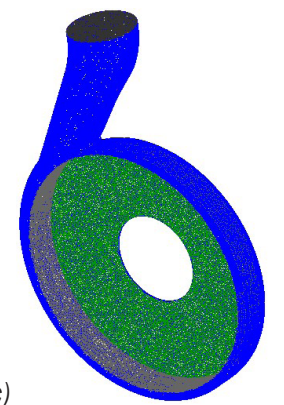

d)

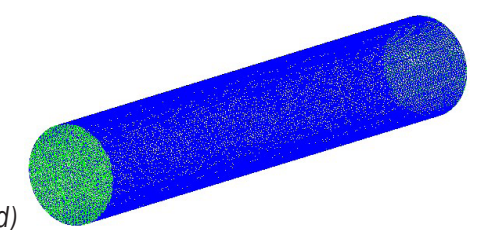


Fig. 6, the head is gradual decline with the number of girds increasing. Relative deviation of head value between case 6 and case 7 was within $\pm 1 \%$, which indicates that it would be little impact on numerical results to increase the number of grids from those of case 6. Taking the computer resources in account and keeping the cost under control, the girds numbers of case 6 were chosen.

\subsection{Boundary Condition and Solution Method}

The velocity inlet and pressure outlet boundary conditions of the model pump were loaded. The hydraulic diameter $D$ of the inlet of the model pump is $65 \mathrm{~mm}$ and the value of turbulence intensity $I$ was calculated directly [18]. Sliding mesh technology was employed to deal with the information transfer between the rotating impeller and the stationary volute. The walls of the model pump were defined as no-slip condition, and the standard wall function was used to solve the low Reynolds number flow near the wall region. Commercial software Fluent based on the finite volume method was amplied to simulate unsteady flow field of model pump. ReNormalisation Group (RNG) $k-\varepsilon$ turbulence model and the SIMPLEC algorithm were chosen to solve the turbulence function and pressure velocity coupling, respectively.

In order to obtain detailed resolution of unsteady flow results of the solid-liquid two-phase flow centrifugal pump, the time step of the unsteady calculation was set as $\Delta t=5.747 \times 10^{-5}$, which indicate that each impeller revolution will be calculated in a time sequence of 360 times steps corresponding to $1^{\circ}$ of the impeller rotation speed. The numerical residual convergence criterion was set as $10^{-5}$ so as to ensure the result to be converged. To obtain a rapid convergence process, steady simulation was first carried out in advance, which was set as the initial condition for transient calculation.

\section{RESULTS AND DISCUSSIONS}

\subsection{Performance Characteristics Analysis}

Comparison of the performance curves of four schemes are shown in Fig. 7. With the increase of flow rate, the heads value of experiment and numerical results for four schemes decrease, and the efficiency value of four schemes increases first and then decreases.

The experiment results show that head and efficiency decrease with the increase of the coating thickness, but their rate of decline increases. Compared with the model pump at nominal flow rate, the head decreases by $3.74 \%, 10.47 \%$ and $24.24 \%$ respectively when the coating thickness coefficients are $K_{1}, K_{2}$ and $K_{3}$, and the efficiency decreases by $3.57 \%, 6.22 \%$ and $14.88 \%$ respectively. It indicates that the coating thickness has great influence on the performance of the model pump. It is mainly because the coating increases the actual thickness of the blade and reduces the flow area of the impeller passage. The performance of the pump is changed. However, the effect of the coating thickness on the performance of the model pump is smaller under low flow rate.
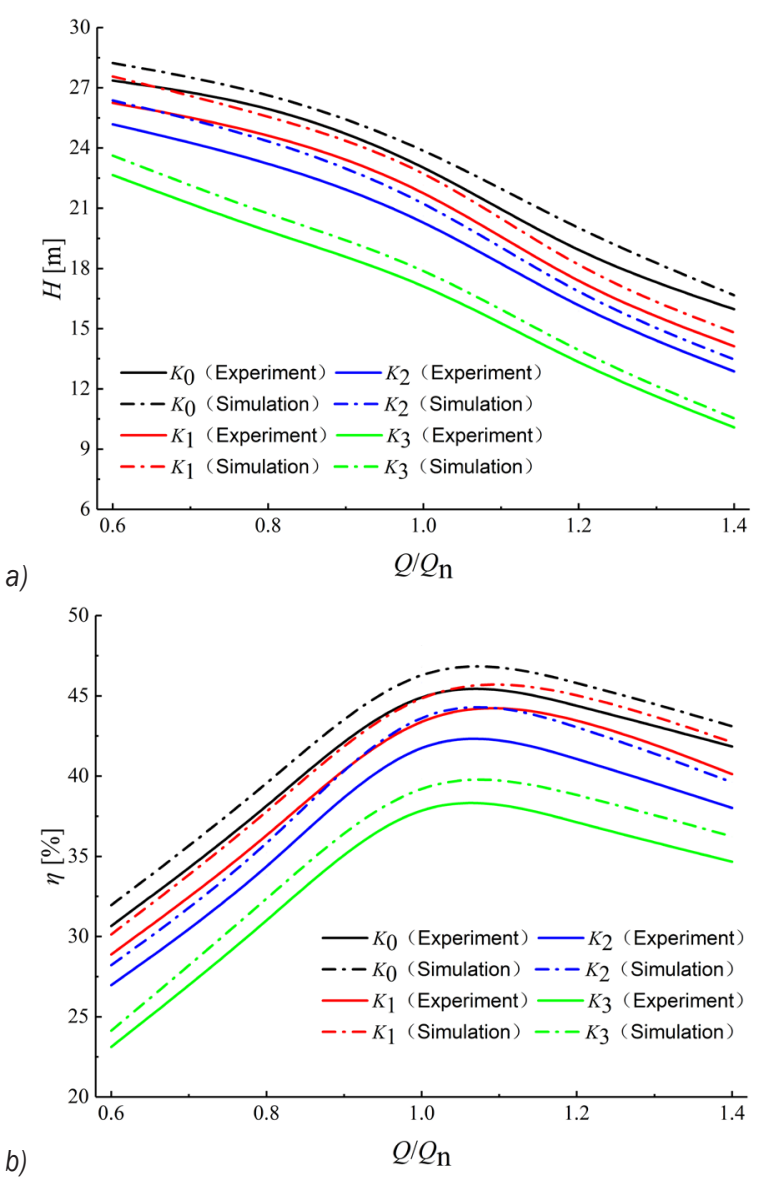

Fig. 7. Mesh sensitivity analysis;

a) head curves; b) efficiency curves

A significant agreement of results between experiment and simulation is captured. Under the same operating condition, the head and efficiency decrease gradually with the increase of the coating thickness. The larger the coating thickness, the greater the decline amplitude. Relative deviations of the head and efficiency at each flow condition are within $5 \%$. 


\subsection{Pressure Pulsation Analysis}

The result of the transient pressure value at the pump outlet was obtained. To make the comparion accurately, the pressure coefficient is introduced:

$$
C_{\mathrm{p}}=\sqrt{\left(\frac{1}{N}\right) \sum\left(p_{\mathrm{i}}-\bar{p}\right)^{2}} /\left(0.5 \rho u_{2}^{2}\right)
$$

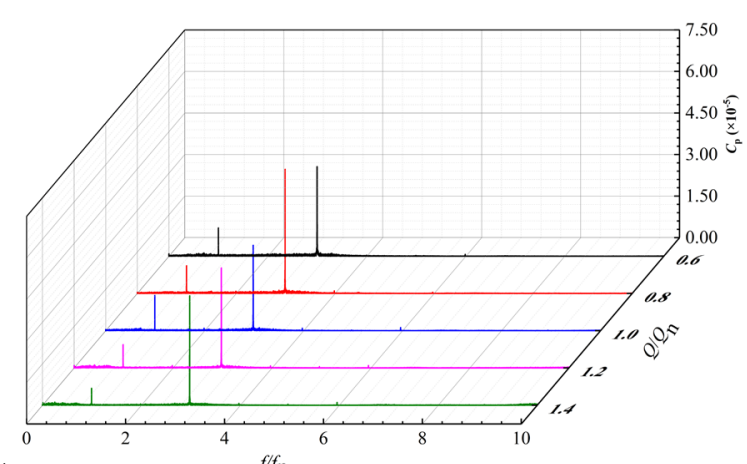

a)

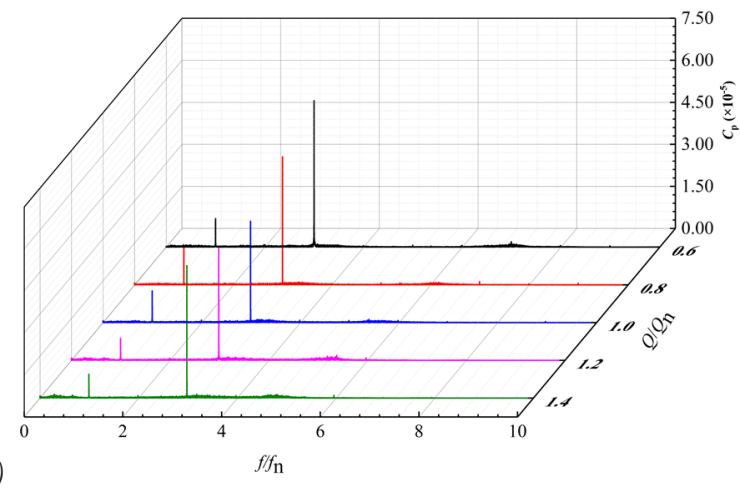

where $p_{\mathrm{i}}$ is the transient static pressure of pump outlet. $\bar{p}$ is the average static pressure for a rotation period of impeller and $u_{2}$ is the circumferential velocity of the impeller.

The experimental results of the pressure fluctuation frequency spectrum of four schemes under different coating thickness are shown in Fig. 8, which

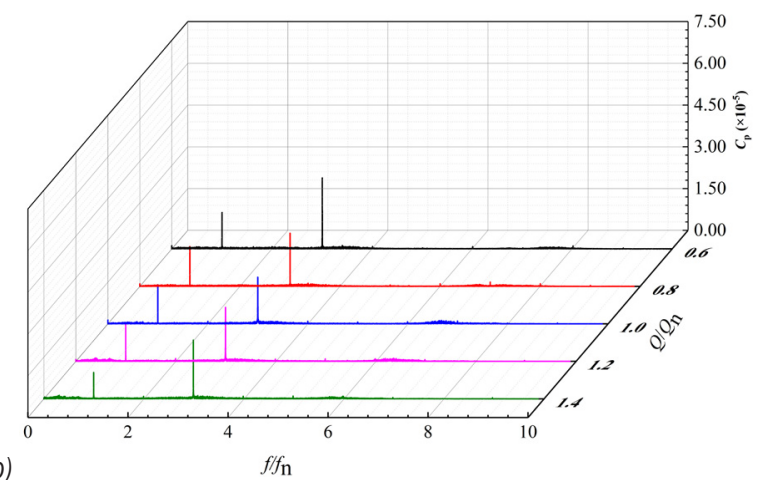

b)

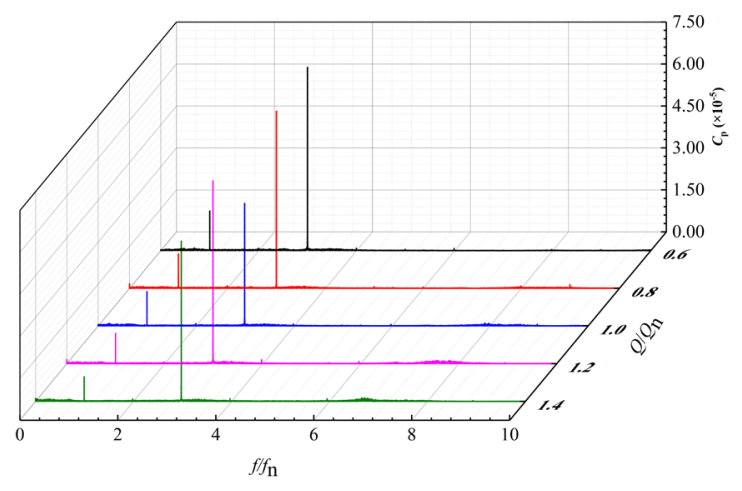

Fig. 8. Pressure fluctuation frequency spectrum; a) KO, b) $K 1$, c) $K 2$, and d) $K 3$

were obtained by fast Fourier transform (FFT). $f$ is the frequency obtained by FFT. $f_{\mathrm{n}}$ is the rotation frequency of the pump shaft.

It can be seen from Fig. 8 that the trend of the pressure fluctuation of the outlet with different coating thickness is basically the same under different operating conditions. The pressure pulsation is found in the low frequency region. Due to the rotor-stator interaction between the impeller and the volute, the main peak value of the pressure fluctuation is at the blade passing frequency. When the coating thickness is the same, the pressure fluctuation coefficient under off-design condition is larger than that under the design condition. It indicates that the flow stability of the off-design condition is worse than that of design condition.

The amplitude of the pressure pulsation frequency spectrum of the numerical simulation after FFT is

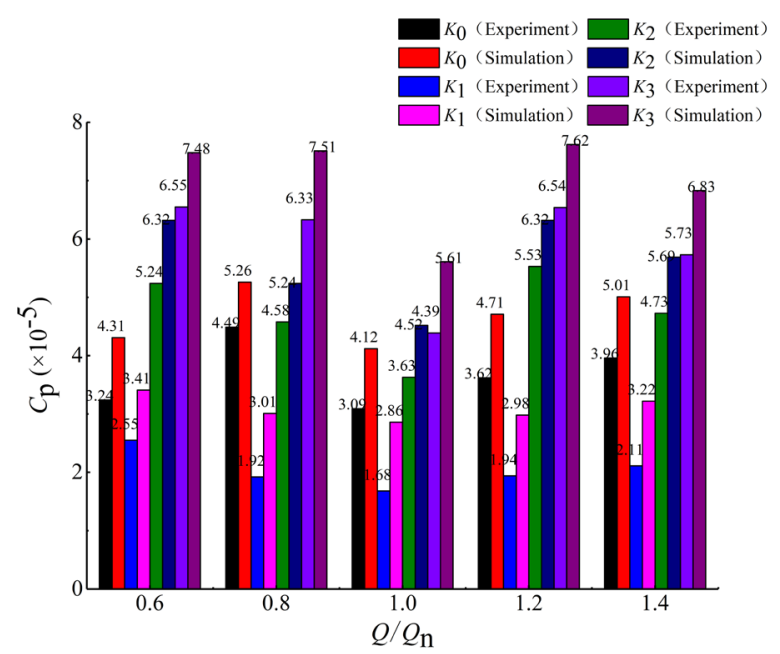

Fig. 9. Peak value of pressure fluctuation between experiment and numerical 
extracted and compared with the experimental value as shown in Fig. 9.

It can be seen from Fig. 9 that the variation trend of the numerical calculation and experimental results under different operation conditions is basically the same with the same coating thickness. The peak value of pressure fluctuation at nominal condition is smaller than that at off-design condition.

The time-domains of the numerical simulation pressure fluctuation under different operating conditions in one impeller rotation cycle with diffident coating thickness are shown in Fig. 10.
It can be seen from Fig. 10 that the variation trend of pressure fluctuation time-domain is identical under different operation conditions with the same coating thickness. Three peaks and three troughs could be captured in a rotation cycle, which is the same as the number of impeller blades. It indicates that rotorstator interaction is the main reason of the pressure pulsation. When the operation condition remains the same, the peak value of the pressure fluctuation of scheme $K_{1}$ is the smallest. With the increase of the coating thickness, the peak value of the pressure fluctuation increases gradually. It indicates that the
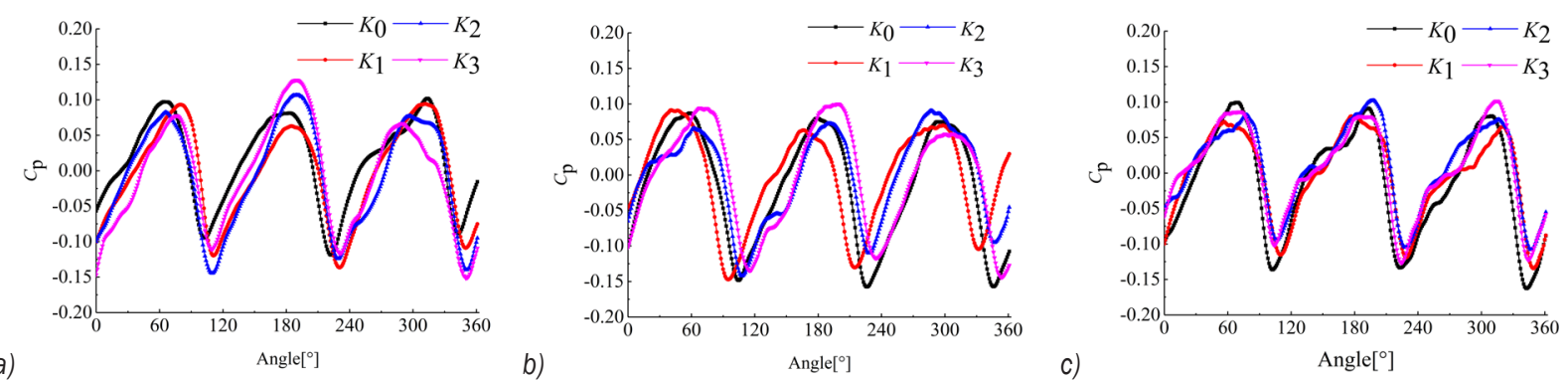

Fig. 10. Time-domain of numerical simulation pressure fluctuation; a) $0.8 Q_{\mathrm{n}}$, b) $1.0 Q_{\mathrm{n}}$, and c) $1.2 Q_{\mathrm{n}}$

a1)
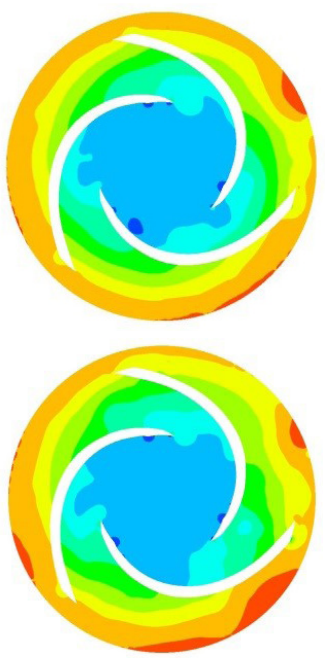

b1)

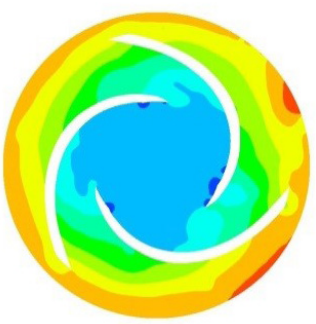

c1)

a2)

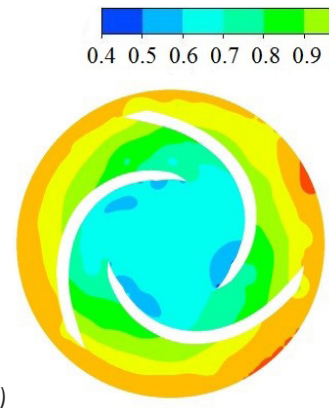

b2)

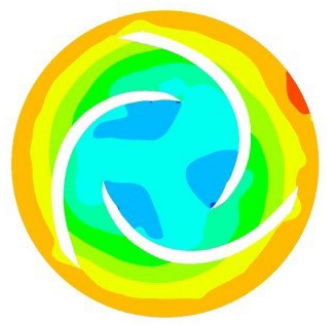

c2)

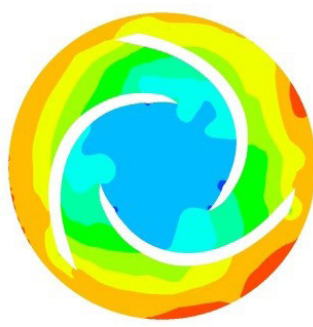

a3)

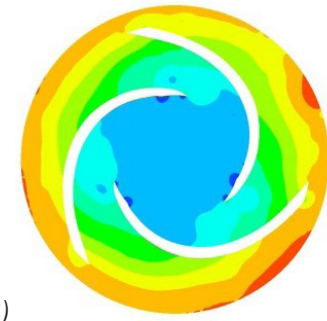

b3)

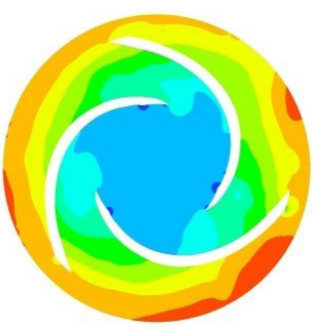

b4)

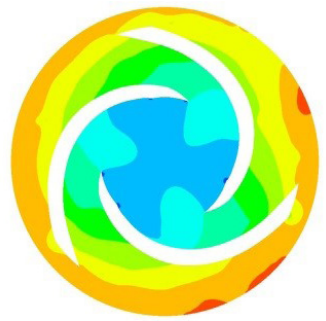

a4)

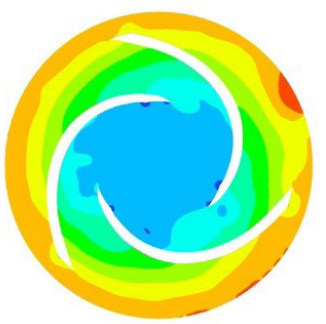

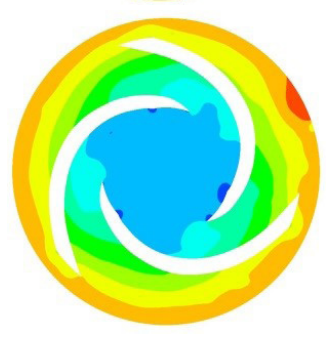

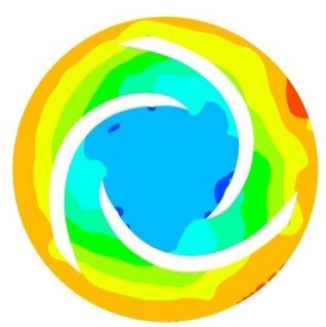

c3)

c4)

Fig. 11. Pressure distributions at the middle section of impeller; a1) $K_{0}, 0.8 Q_{\mathrm{n}}$; a2) $K_{1}, 0.8 Q_{\mathrm{n}}$; a3) $K_{2}, 0.8 Q_{\mathrm{n}}$; a4) $K_{3}, 0.8 Q_{\mathrm{n}}$; b1) $K_{0}, 1.0 Q_{\mathrm{n}}$; b2) $K_{1}, 1.0 Q_{\mathrm{n}}$; b3) $K_{2}, 1.0 Q_{\mathrm{n}}$; b4) $K_{3}, 1.0 Q_{\mathrm{n}}$; c1) $K_{0}, 1.2 Q_{\mathrm{n}}$; c2) $K_{1}, 1.2 Q_{\mathrm{n}}$; c3) $K_{2}, 1.2 Q_{\mathrm{n}}$; c4) $K_{3}, 1.2 Q_{\mathrm{n}}$ 
coating thickness of blade will increase the instability of pump outlet flow. The thickness of the blade will be increased after the coating treatment, which results in the enhanced rotor-stator interaction between the impeller and the tongue of volute.

\subsection{Internal Flow Analysis}

Fig. 11 shows the pressure distributions at the middle section of the impeller under the conditions of $0.8 Q_{\mathrm{n}}$, $1.0 Q_{\mathrm{n}}$ and $1.2 Q_{\mathrm{n}}$, respectively.

As can be easily seen in Fig. 11. The variation trend of the static pressure is basically the same under different operation conditions with the same coating thickness. Low pressure area at the inlet of the impeller is captured. With the increase of the flow rate, the range of the low pressure area increases gradually.

With the increase of the coating thickness, the pressure at the inlet of the impeller increases first and then decreases under high flow rate and design conditions, which is related to the change of the relative flow angle of fluid. It is clarified that the coating thickness on impeller should be within a certain range, and the static pressure at the inlet of the impeller can be increased relatively within this range.

Based on the above analysis, it is considered that the static pressure at the inlet of the impeller can be increased when the coating thickness coefficient is $K_{1} \sim K_{2}$ under the condition of clear water.

Fig. 12 mainly focus on the relative velocity and streamline diagram at the middle section of the impeller under the conditions of $0.8 Q_{\mathrm{n}}, 1.0 Q_{\mathrm{n}}$ and $1.2 Q_{\mathrm{n}}$ respectively.

It can be seen from Fig. 12 that under the same operation condition, different sizes of low speed zones in the impeller runner with different coating thickness are caught, and the low speed zones with uneven distribution will lead to the instability of flow in the runner. With the increase of the coating thickness, the area of the low-speed area in the impeller runner decreases first and then increases. It is shown that proper coating thickness on blades can reduce the a1)

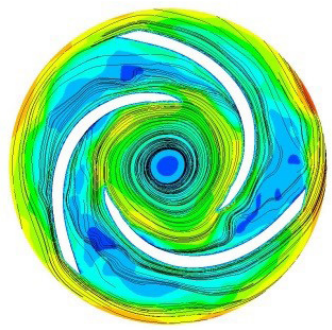

b1)

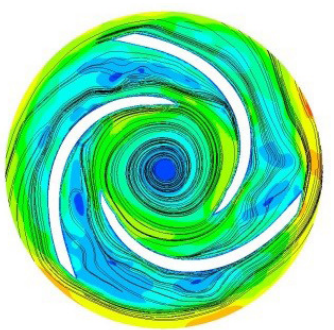

c1)

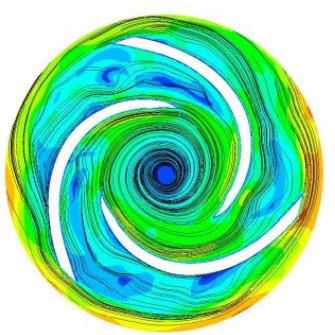

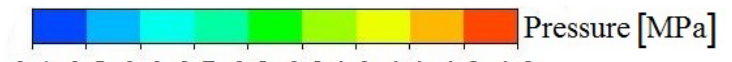

$\begin{array}{llllllllll}0.4 & 0.5 & 0.6 & 0.7 & 0.8 & 0.9 & 1.0 & 1.1 & 1.2 & 1.3\end{array}$

a2)

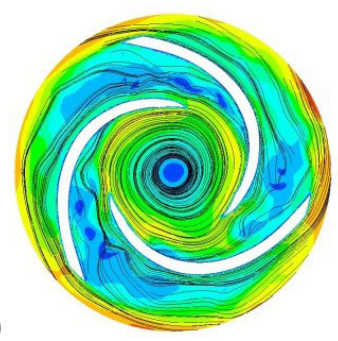

a3)
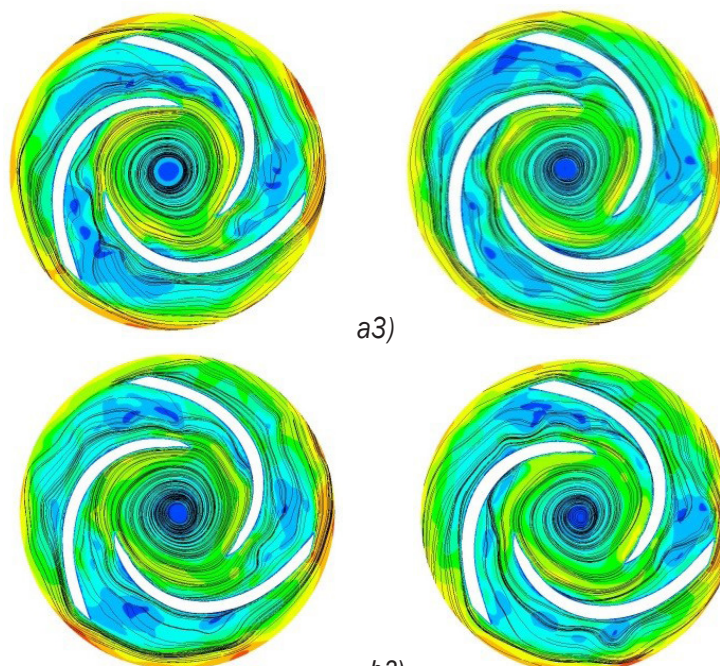

b2)

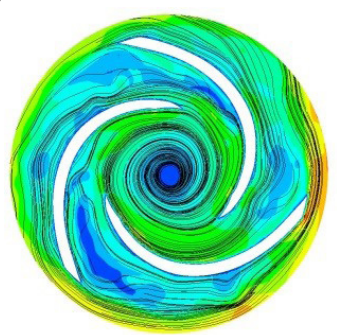

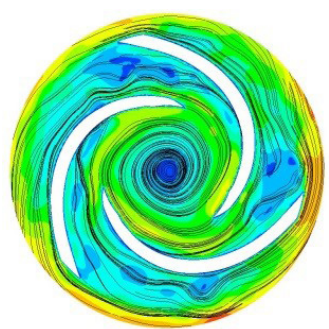

b3)

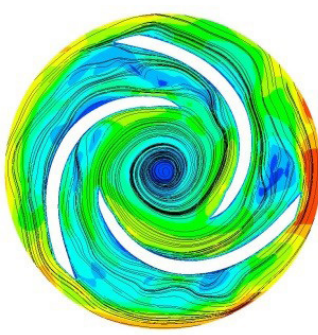

a4)

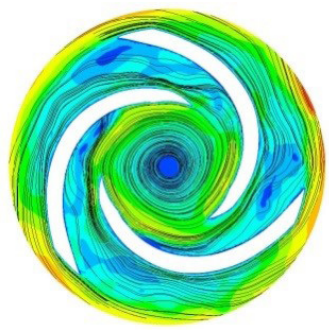

b4)

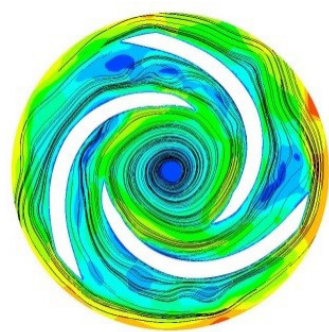

c4)

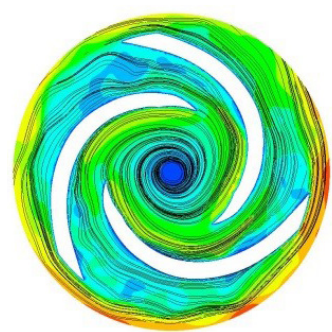

Fig. 12. Velocity and streamline diagram at middle section of impeller; ; a1) $K_{0}, 0.8 Q_{\mathrm{n}}$; a2) $K_{1}, 0.8 Q_{\mathrm{n}}$; a3) $K_{2}, 0.8 Q_{\mathrm{n}}$; a4) $K_{3}, 0.8 Q_{\mathrm{n}}$; b1) $K_{0}, 1.0 Q_{\mathrm{n}}$; b2) $K_{1}, 1.0 Q_{\mathrm{n}}$; b3) $K_{2}, 1.0 Q_{\mathrm{n}}$; b4) $K_{3}, 1.0 Q_{\mathrm{n}}$; c1) $K_{0}, 1.2 Q_{\mathrm{n}}$; c2) $K_{1}, 1.2 Q_{\mathrm{n}}$; c3) $K_{2}, 1.2 Q_{\mathrm{n}}$; c4) $K_{3}, 1.2 Q_{\mathrm{n}}$ 
instability in the runner, which is consistent with the analysis of pressure fluctuation. The low speed zone decreases gradually with the increase of the flow rate under the same thickness.

As can be shown in Fig. 12 approximate circular vortex could be found near the impeller inlet of four scheme in each operation condition, and the vortex velocity is small. It is since that the model pump impeller does not have a front cover, and a vortex is formed by the impeller rotating. Higher liquid velocity outside the vortex at the impeller inlet results in lower pressure here, which is consistent with the static pressure analysis above. Under the same operation condition, the circular vortex at the inlet decreases with the increase of the coating thickness, which leads to the increase of the inlet velocity of the impeller. The streamline distribution in the channel gradually changes from uniform to near the impeller working face, and the streamline near the low pressure region has similar vortex. When the coating thickness is the same, the range of the low pressure zone under the design condition is smaller than that under nondesigned condition, and the streamline distribution is more uniform, which indicates that the coating treatment does not change the inherent property of the model pump.

\subsection{Radial Force Analysis of Impeller}

Fig. 13 is the radial force distribution of the pump impeller for four coatingschemes.

It can be seen from Fig. 13 that the radial force of the impeller varies periodically with time under various working conditions and distributes symmetrically in a triangle, which is the same as the number of blades. Under the same operation condition, the radial force increases with the increase of the coating thickness. It is because that the flow area decreases after the coating treatment, which result in the change of the flow stability in the passage. The radial force of impeller is minimum at the designed condition under the same coating thickness.

Fig. 14 is the histogram of the radial force amplitude of the pump impeller with four coating thickness models. Compared with the coating thickness coefficient $K_{0}$, the radial force of the impeller increases by $14.28 \%, 22.06 \%$ and $32.31 \%$ when the coating thickness coefficients are $K_{1}, K_{2}$ and $K_{3}$, respectively.
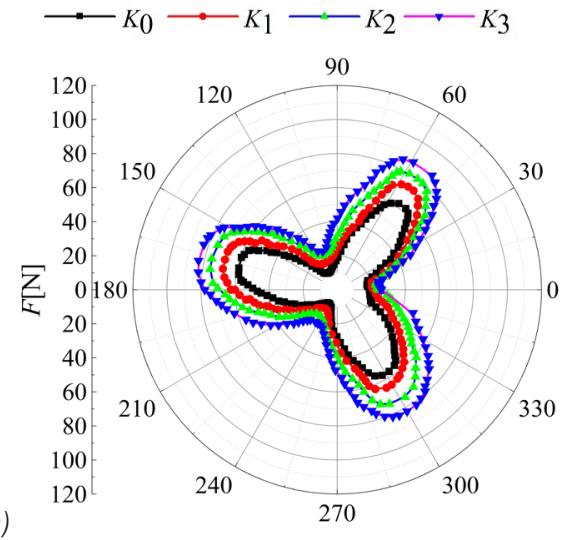

a)
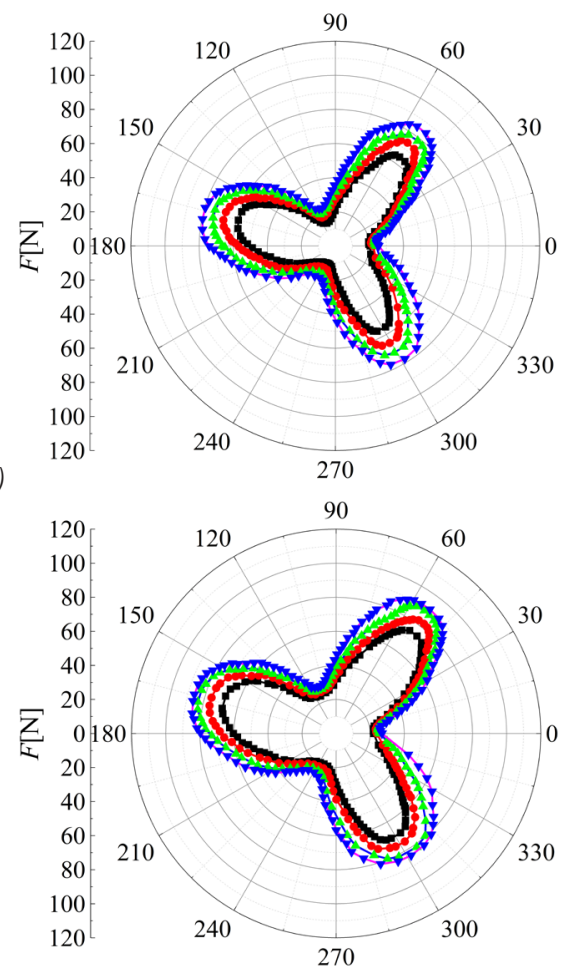

Fig. 13. Radial force of impeller; a) $0.8 Q_{\mathrm{n}}$; b) $1.0 Q_{\mathrm{n}}$; c) $1.2 Q_{\mathrm{n}}$

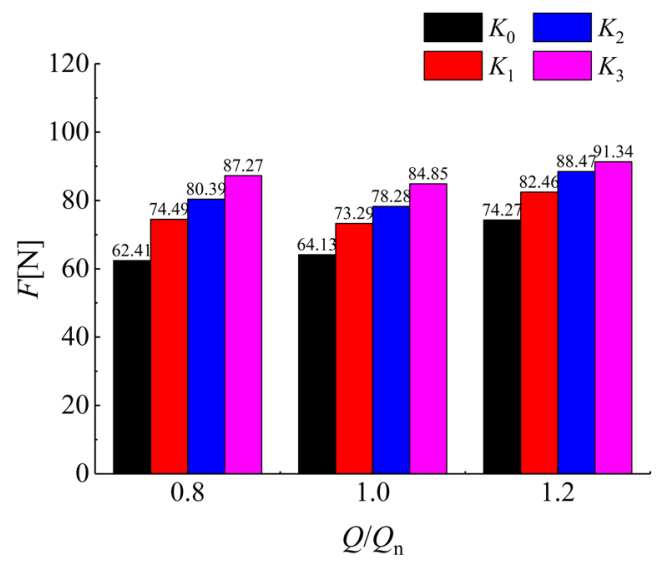

Fig. 14. Comparison of radial force for four schemes 


\section{CONCLUSIONS}

In this paper, polyurethane coating with good wear resistance was sprayed on the pressure surface of the impeller blade. The performance, pressure fluctuation, internal flow and radial force of four kinds of coating schemes under water condition were studied by experiment and numerical simulation.

1) The experimental results show that the head and efficiency decrease with the increase of the coating thickness, and the decline amplitude is proportional to the coating thickness

2) The outlet pressure pulsation is concentrated in the low frequency region, and the main peak value of pressure fluctuation is at the blade frequency. Under the design condition, the amplitude and peak value of pressure fluctuation are the smallest when the coating thickness coefficient is $K_{1}$. The peak value of pressure fluctuation increases gradually with the coating thickness increasing.

3) There is a large area of low pressure at each impeller inlet. As the coating thickness increases, the pressure at the impeller inlet increases first and then decreases.

4) There are different sizes of low velocity zones in impeller flow channel under each coating thickness. With the increase of the coating thickness, the streamline in the channel becomes more and more inhomogeneous, and the streamline distribution in the impeller changes from uniform to near the impeller pressure surface.

5) The radial force of the impeller increases with the increase of coating thickness, and the radial force of impeller distributes symmetrically in triangle, which is the same as the number of blades.

\section{ACKNOWLEDGEMENTS}

The authors would like to thank the support by the National Key Research and Development Program of China (2017YFC0804107), the National Natural Science Foundation of China (51779106, 51609164), Ministry of Education, Xihua University (szjj2016068), Jiangsu top six talent summit project (GDZB017), the Natural Science Foundation of Jiangsu Province (Grant No. BK20160574).

\section{NOMENCLATURES}

$Q_{\text {n }}$ nominal flow rate, $\left[\mathrm{m}^{3} / \mathrm{h}\right]$

$H$ head, [m]

$n$ rotational speed, [rpm] $n_{\mathrm{s}} \quad$ specific speeds, $n_{\mathrm{s}}=3.65 n Q^{1 / 2} / \mathrm{H}^{3 / 4}$

$\eta \quad$ efficiency

$u_{2}$ impeller circumferential velocity, $[\mathrm{m} / \mathrm{s}]$

$D_{1}$ impeller inlet diameter, [mm]

$D_{2}$ impeller outlet diameter, [mm]

$D_{3}$ inlet diameters of volute, $[\mathrm{mm}]$

$D_{4}$ diffuser outlet diameter, [mm]

$b_{3}$ inlet width of volute, [mm]

$z \quad$ blade number of impeller

$\bar{p}$ average static pressure in a rotation period of impeller, $[\mathrm{Pa}]$

$\delta \quad$ thickness of blade, [mm]

$\delta_{\mathrm{i}} \quad$ coating thickness

$\rho$ density, $\left[\mathrm{kg} / \mathrm{m}^{3}\right]$

$b \quad$ outlet impeller width, [mm]

$f_{\mathrm{n}}$ rotation frequency of pump shaft, $[\mathrm{Hz}]$

$K_{i}$ ratio of coating thickness

$C_{\mathrm{p}}$ time-dependent nondimensional pressure coefficient

\section{REFERENCES}

[1] Noon, A.A., Kim, M.-H. (2016). Erosion wear on centrifugal pump casing due to slurry flow. Wear, vol. 364-365, p. 103111, D0I:10.1016/j.wear.2016.07.005.

[2] Lai, F., Zhu, X., Xu, X., Li, G. (2018). Erosion wear and performance simulation of centrifugal pump for solid-liquid flow. ASME Power Conference collocated with the ASME $12^{\text {th }}$ International Conference on Energy Sustainability and the ASME Nuclear Forum, p. V002T10A001-V002T10A001, DOI:10.1115/POWER2018-7151.

[3] Sharma, A.K., (2008). Numerical Study of Erosion Wear on Centrifugal Slurry Pump. MSc thesis, Thapar University, Patiala.

[4] Bross, S., Addie, G. (2002). Prediction of impeller nose wear behaviour in centrifugal slurry pumps. Experimental Thermal and Fluid Science, vol. 26, no. 6-7, p. 841-849, Dol:10.1016/ S0894-1777(02)00174-7.

[5] Wu, B., Wang, X.-L., Liu, H., Xu, H.-L. (2015). Numerical simulation and analysis of solid-liquid two-phase threedimensional unsteady flow in centrifugal slurry pump. Journal of Central South University, vol. 22, no. 8, p. 3008-3016, DOl:10.1007/s11771-015-2837-7.

[6] Shi, B.C., Wei, J., Zhang, Y. (2017). A novel experimental facility for measuring internal flow of Solid-liquid two-phase flow in a centrifugal pump by PIV. International Journal of Multiphase Flow, vol. 89, p. 266-276, D0l:10.1016/j. ijmultiphaseflow.2016. 11.002.

[7] Ning, C., Wang, Y. (2016). Performance analysis on solidliquid mixed flow in a centrifugal pump. IOP Conference Series: Materials Science and Engineering, vol. 129, no. 1, p. 012062, D0I:10.1088/1757-899X/129/1/012062.

[8] Wang, Z., Qian, Z. (2017). Effects of concentration and size of silt particles on the performance of a double-suction centrifugal pump. Energy, vol. 123, p. 36-46, D0I:10.1016/j. energy.2017.01.142. 
[9] Mishra, P., Ein-Mozaffari, F. (2017). Using computational fluid dynamics to analyze the performance of the Maxblend impeller in solid-liquid mixing operations. International Journal of Multiphase Flow, vol. 91, p. 194-207, D0l:10.1016/j. ijmultiphaseflow.2017.01.009.

[10] Cando, E., Yu, A., Zhu, L., Liu, J., Lu, L., Hidalgo, V., Luo, X.W. (2017). Unsteady numerical analysis of the liquid-solid twophase flow around a step using Eulerian-Lagrangian and the filter-based RANS method. Journal of Mechanical Science and Technology, vol. 31, no. 6, p. 2781-2790, Dol:10.1007/ s12206-017-0521-6.

[11] Zhao, W.G., Zhao, G.S. (2018). Numerical investigation on the transient characteristics of sediment-laden two-phase flow in a centrifugal pump. Journal of Mechanical Science and Technology, vol. 32, no. 1, p. 167-176, Dol:10.1007/s12206017-1218-6.

[12] Golru, S.S., Attar, M.M., Ramezanzadeh, B. (2014). Studying the influence of nano-Al203, particles on the corrosion performance and hydrolytic degradation resistance of an epoxy/polyamide coating on AA-1050. Progress in Organic Coatings, vol. 77 , no. 9, p. 1391-1399, D0l:10.1016/j. porgcoat.2014.04.017.

[13] Esteves, M., Ramalho, A., Ferreira, J.A.M., Nobre, J.P. (2013). Tribological and mechanical behaviour of epoxy/nanoclay composites. Tribology Letters, vol. 52, no. 1, p. 1-10, DOI:10.1007/s11249-013-0174-2.
[14] Chairman, C.A., Kumaresh Babu, S.P. (2013). Mechanical and abrasive wear behavior of glass and basalt fabric-reinforced epoxy composites. Journal of Applied Polymer Science, vol. 130, no. 1, p. 120-130, DOI:10.1002/app.39154.

[15] Jin, J., Rafiq, R., Gill, Y.Q., Song, M. (2013). Preparation and characterization of high performance of graphene/nylon nanocomposites. European Polymer Journal, vol. 49, no. 9, p. 2617-2626, D0l:10.1016/j.eurpolymj.2013.06.004.

[16] Kim, B.K., Lee, J.C. (1996). Waterborne polyurethanes and their properties. Journal of Polymer Science Part A: Polymer Chemistry, vol. 34, no. 6, p. 1095-1104, D0I:10.1002/(SICI)1099-0518(19960430)34:6<1095::AIDPOLA19>3.0.C0;2-2.

[17] Fu, J., Chen, T., Wang, M., Yang, N., Li, S., Wang, Y., Liu, X. (2013). Acid and alkaline dual stimuli-responsive mechanized hollow mesoporous silica nanoparticles as smart nanocontainers for intelligent anticorrosion coatings. ACS Nano, vol. 7, no. 12, p. 11397-11408, Dol:10.1021/ nn4053233.

[18] Zhang, N., Yang, M., Gao, B., Li, Z., Ni, D. (2016). Investigation of rotor-stator interaction and flow unsteadiness in a low specific speed centrifugal pump. Strojniški vestnik Journal of Mechanical Engineering, vol. 62, no. 1, p. 21-33, D0I:10.5545/sv-jme.2015.2859. 